\title{
INTEGRATING A LOW-COST MEMS IMU INTO A LASER-BASED SLAM FOR INDOOR MOBILE MAPPING
}

\author{
S. Karam ${ }^{1, *}$, V. Lehtola ${ }^{1}$, G. Vosselman ${ }^{1}$ \\ ${ }^{1}$ Dept. of Earth Observation Science, Faculty ITC, University of Twente, 7514 AE Enschede, The Netherlands \\ (s.karam, v.v.lehtola, george.vosselman)@utwente.nl
}

\section{Commission II}

KEY WORDS: Indoor mapping, mobile laser scanning, SLAM, IMU, integration, point clouds, 2D laser scanner

\begin{abstract}
:
Indoor mapping techniques are highly important in many applications, such as human navigation and indoor modelling. As satellite positioning systems do not work in indoor applications, several alternative navigational sensors and methods have been used to provide accurate indoor positioning for mapping purposes, such as inertial measurement units (IMUs) and simultaneous localisation and mapping algorithms (SLAM). In this paper, we investigate the benefits that the integration of a low-cost microelectromechanical system (MEMS) IMU can bring to a feature-based SLAM algorithm. Specifically, we utilize IMU data to predict the pose of our backpack indoor mobile mapping system to improve the SLAM algorithm. The experimental results show that using the proposed IMU integration method leads into a more robust data association between the measured points and the model planes. Notably, the number of points that are assigned to the model planes is increased, and the root mean square error (RMSE) of the residuals, i.e. distances between these measured points and the model planes, is decreased significantly from $1.8 \mathrm{~cm}$ to $1.3 \mathrm{~cm}$.
\end{abstract}

\section{INTRODUCTION}

There is a need for indoor mapping in many important applications, such as the mapping of hazardous sites, indoor navigation, disaster management, location-based services, and virtual reality displays. Since digital maps of public buildings (airports, hospitals, train stations, and so forth) are a prerequisite for navigating their interiors, there is a trend towards the development of geospatial indoor applications (Norris, 2013). In order to avoid the time-consuming and intense efforts that static mapping systems require to map building interiors, there has been increasing interest in indoor mobile mapping systems in recent years (Lehtola et al., 2017). As GNSS-based systems do not work indoors, several alternative navigational methods and sensors have been used to provide accurate indoor positioning for mapping purposes, such as simultaneous localisation and mapping algorithms (SLAMs) and inertial measurement units (IMUs). The essential solution methods and computational complexity of the SLAM problem are described by DurrantWhyte and Bailey (2006a, 2006b).

SLAM has become a key technology in indoor mapping applications, and a wide variety of different SLAM algorithms have been proposed. These algorithms are based on data from cameras (Henry et al., 2014), laser scanners like HectorSLAM (Kohlbrecher et al., 2011) and Gmapping (Grisetti et al., 2007), (Lehtola et al., 2016; Wen et al., 2016)) or both (Liu et al., 2010; Naikal et al., 2009). Surveys, as conducted by Maximov (2013), have shown that the integration of multiple sources of navigational information improves the accuracy of a navigation system. IMUs are one of the most commonly used navigational data sources in attitude (Hyyti et al., 2015; Makni et al., 2014) and pose estimation methods (Feliz et al., 2009). In addition to being relatively inexpensive, the MEMS-based IMUs are relatively small in size, lightweight, and low in power consumption; as such, they are widely integrated into indoor navigation systems. For example, an IMU is fused with a Hokuyo scanner on unmanned aerial vehicles (UAVs) (Kumar et al.,
2017) and with a Velodyne scanner and panorama camera on backpack platforms (Blaser et al., 2019).

Many works have integrated visual and inertial sensors within indoor SLAM algorithms (Chow et al., 2014; Concha et al., 2016; García et al., 2016; Leutenegger et al., 2015; Wang et al., 2018). However, the camera-based SLAM algorithms fail in textureless or repetitive environments because those algorithms search for similar features in consecutive images. Moreover, the light conditions in indoor environments are sometimes not good enough for capturing high-quality images.

In our previous work (Karam et al., 2019), we built our featurebased SLAM algorithm based on three Hokuyo laser scanners. In this paper, we investigate the benefits obtained from integrating a low-cost microelectromechanical system (MEMS) IMU into this SLAM. Our effort is characterized by that we try to keep our system as inexpensive as possible by using less expensive LiDAR sensors with the low-cost IMU. All the involved sensors are mounted on a backpack platform, which provides more freedom than UAVs in terms of the weight of the mounted components.

An IMU has strengths and weaknesses. Using only the IMU to navigate, the so-called dead reckoning leads to the drift of the predicted position from the physical one due to biases in the sensor observations. The IMU can provide reliable estimations of positions and attitudes for a short while, but its reliability decreases over time. In this work, we seek to exploit the strength of the IMU in measuring short-term pose changes and improving the pose prediction, thereby improving the data association robustness of the SLAM method.

The remainder of this paper is organized as follows: Section 2 presents the related works. Section 3 describes our backpack mapping system and the laser-based SLAM algorithm. Then we elaborate the strategy used in the IMU-SLAM integration. In

\footnotetext{
* Corresponding author
} 
Section 4, we provide a brief overview of the datasets used to investigate this mapping technique. The analysis of the IMU performance is presented in Section 5. Section 6 discusses the results obtained from integrating the IMU with the laser-based SLAM. Finally, the paper draws conclusions in Section 7.

\section{RELATED WORK}

IMUs are widely used in indoor navigation and mapping systems. In addition to the works mentioned in the introduction, there are some specific ones that are closely related. For instance, an IMU is combined with one Hokuyo scanner and utilized for position estimation in 3D hand-held laser scanning system-ZEB-REVO (GeoSLAM, 2018) — evolved by GeoSLAM company. Blaser et al. (2019) incorporated MEMS IMU with two Velodyne VLP-16 laser scanners and one panorama camera in a portable mobile mapping system. The fusion of IMU and scanners in their SLAM is exploited to orient the camera in indoor environments. The Leica Pegasus backpack system also integrates a dual Velodyne VLP-16 scanner with a high precision IMU for indoor mapping (Leica Geosystems, 2016). In addition, Lauterbach et al. (2015) presented a backpack mapping system equipped with 2D (SICK LMS 100) and 3D (Riegl VZ-400) laser scanners, and an IMU (Phidgets 1044). Two SLAM algorithms (3DOF HectorSLAM and 6DOF semi-rigid SLAM) execute successively, with the output of one being the input of the other. The first one, HectorSLAM, uses the data of SICK scanner and an IMU for initial trajectory estimation. The semi-rigid SLAM exploits this initial pose estimation to align point clouds captured by the 3D scanner. The integration of IMU data can also be utilised to increase the degrees of freedom (DOF) of a mobile system. For instance, Wen et al. (2016) extended a horizontal laser-based 2D SLAM using rotations captured by an IMU to obtain a 3D (6DOF) pose and improve the accuracy of the 3D map. Recently, Velas et al. (2019) proposed another mobile backpack solution that combines a pair of Velodyne scanners with IMU and satellite positioning. This makes the system capable to work outdoors as well. The IMU is utilized in indoor applications to align the horizontal planes, such as floor and ceiling, with the XY plane in the 3D model.

\section{LASER-BASED SLAM AND IMU INTEGRATION}

In the following section, we first describe our mobile mapping system and the employed laser-based SLAM algorithm. Then we introduce the methodology used to integrate IMU with SLAM.

\subsection{System Components}

Our backpack indoor mobile mapping system consists of three time-of-flight (TOF) scanners (Hokuyo UTM-30LX) and one Xsens MEMS IMU. The top scanner is horizontally positioned and mounted on the top of the backpack system, while the other two scanners are tilted and mounted to the right and left of the top one, as shown in Figure 1. The IMU is horizontally positioned and mounted underneath the top scanner. A laptop running Ubuntu 16.04.X and the robot operation system (ROS) is used to communicate with all the mounted sensors and visualize the captured data over time.

\subsection{Coordinate Systems and Registration Process}

Figure 1 shows the various coordinate systems in our backpack mapping system. In order to accurately fuse data from multiple sensors, their individual coordinate systems must be transformed into a unified coordinate system called the frame coordinate system (f). We adopt the coordinate system of the top scanner as the frame coordinate system. As described in our previous work (Karam et al., 2019), the two tilted scanners are registered in this frame coordinate system. For the relative rotation of the IMU with respect to the frame coordinate system, the z-axes of both the IMU and frame are assumed to be aligned by design. For full alignment, the IMU sensor system (s) need to be rotated around the z-axis through a $90^{\circ}$ angle in a clockwise direction $\left(R_{S}^{f}\right)$.

As the frame system is constantly moving, we need to define a fixed coordinate system in which the final 3D model will be defined. Our fixed model system $(\mathrm{m})$ is established from the first scans of the three scanners, as described by Vosselman (2014). The moving frame system is registered in this model coordinate system over time $t$ using six transformation parameters, namely, three rotation parameters $\left(\omega_{f_{\mathrm{t}}}^{m}, \varphi_{f_{\mathrm{t}}}^{m}, \kappa_{f_{\mathrm{t}}}^{m}\right)$ form the rotation matrix $R_{f_{t}}^{m}$ and three translation parameters $\left(X_{f_{\mathrm{t}}}^{m}, Y_{f_{t}}^{m}, Z_{f_{\mathrm{t}}}^{m}\right)$ form the translation vector $T_{f_{t}}^{m}$. These transformation parameters are estimated within the laser-based SLAM.

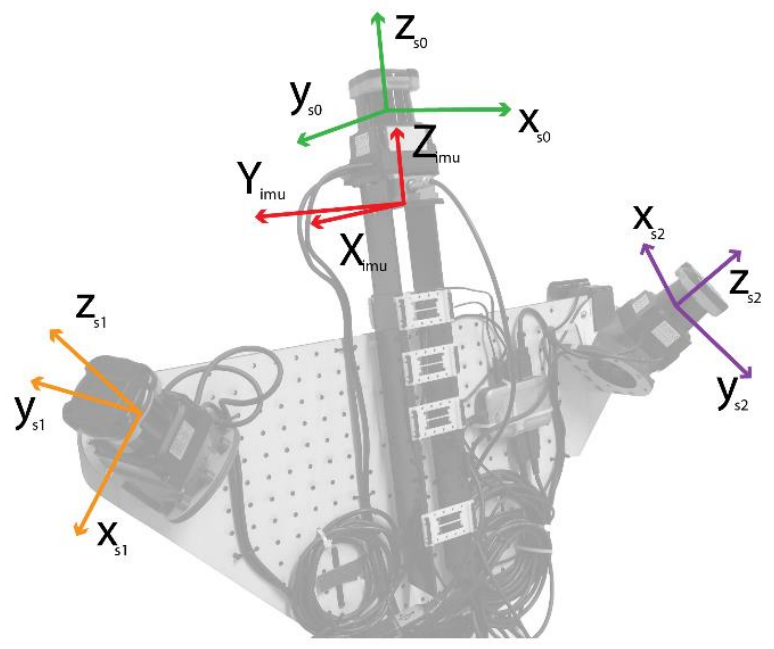

Figure 1. The backpack system with coordinate systems plotted for all four mounted sensors: the three scanners $S_{0}, S_{1}$, and $S_{2}$, and the Xsens IMU (below $\mathrm{S}_{0}$ ).

\subsection{Laser-based SLAM}

Laser-based SLAM is a feature-based SLAM with 6DOF, three position $\left(T_{f_{t}}^{m}\right)$ and three attitude $\left(R_{f_{t}}^{m}\right)$ parameters. As the scanning frequency of the Hokuyo model used is $40 \mathrm{HZ}$, each scanner records one scanline within a local time window of 25 ms. Our SLAM senses planar features, horizontal and vertical, in the mapped environment through the linear segments that are detected in the single scanlines. We model the frame pose parameters as a function of time using B-splines and define each plane by its normal vector and distance to the origin in the model coordinate system.

The solution of SLAM goes through two consecutive laser-based estimation processes, a local pose spline estimation involving the data captured during only 75-100 ms, and a global adjustment. The methods are explained in detail in Karam et al. (2019). In this paper, we focus on the local spline estimation process in which the pose is predicted and the data association is tested. This estimation is based on the laser observations of only three to four scanlines from each of the scanners. The algorithm tries to establish the association between the linear segments in the three newly captured scanlines within the local window and the 
previously reconstructed planes. This test is based on the predicted pose resulting from a linear extrapolation of the locally estimated splines. It uses a distance threshold to decide whether a segment should be associated with a previously reconstructed plane or whether a new horizontal/vertical plane needs to be instantiated. When the whole dataset is processed locally, the SLAM runs a final adjustment process that estimates not only the trajectory parameters but also the parameters of all the reconstructed planes in the model coordinate system.

\subsection{IMU-based Pose Prediction}

Here, we consider an Xsens MEMS IMU that is a combination of three-axial accelerometers used to measure dynamic acceleration and gravity and three-axial gyroscopes used to measure angular velocity. The accelerations and angular velocities are collected with a sampling frequency of $200 \mathrm{HZ}$; thus, there are few IMU measurements taken within each local window of one scanline. As an alternative to linear extrapolation, those measurements are utilized to provide a more reliable prediction of the system's position and attitude, as described in the following subsections.

We assume that the local window, for which we want to predict the pose, starts at $t_{\text {start }}$ and ends at $t_{\text {end }}$. The IMU measurements are the angular velocities $\left(\dot{\omega}_{i m u}^{s_{t_{i}}}, \dot{\varphi}_{i m u}^{s_{t_{i}}}, \dot{\kappa}_{i m u}^{s_{t_{i}}}\right)$ and accelerations $\left(\ddot{X}_{i m u}^{s_{t_{i}}}, \ddot{Y}_{i m u}^{s_{t_{i}}}, \ddot{Z}_{i m u}^{s_{t_{i}}}\right.$ ) observed at time $t_{i}$ in the IMU sensor $\operatorname{system}(\mathrm{s})$, where $i=1,2, \ldots, \mathrm{n}$. We select $t_{1}$ as the timestamp of the last IMU measurement before $t_{\text {start }}$ and $t_{n}$ as the timestamp of the first IMU measurement after $t_{\mathrm{end}}$.

The following pose parameters are known at $t_{1}$ from the SLAM estimation process that ran before:

- The attitude parameters $\left(\omega_{f_{t_{1, \text { slam }}}}^{m}, \varphi_{f_{t_{1 \text { slam }}}}^{m}, \kappa_{f_{t_{1 \text { slam }}}}^{m}\right)$ form the rotation matrix $R_{f_{t_{1, \text { slam }}}^{m}}$ from the frame coordinate system to the world coordinate system at $t_{1}$.

$$
R_{f t_{1, \text { slam }}}^{m}=R_{1}\left(\omega_{f_{t_{1, \text { slam }}}^{m}}^{m}\right) R_{2}\left(\varphi_{f_{t_{1, \text { slam }}}^{m}}^{m}\right) R_{3}\left(\kappa_{f_{t_{1, \text { slam }}}^{m}}^{m}\right)
$$

- The position parameters $\left(X_{f_{t_{1, \text { slam }}}}^{m}, Y_{f_{t_{1, \text { slam }}}}^{m}, Z_{f_{t_{1, \text { slam }}}}^{m}\right)$ form the translation vector $T_{f_{1, \text { slam }}}^{m}$ from the frame coordinate system to the world coordinate system at $t_{1}$.

$$
T_{f_{t_{1, \text { slam }}}^{m}}^{m}=\left(X_{f_{t_{1, \text { slam }}}^{m}}^{m} \quad Y_{f_{t_{1, \text { slam }}}^{m}}^{m} \quad Z_{f_{t_{1, \text { slam }}}^{m}}^{m}\right)^{T}
$$

- The approximate velocity of the frame in the model coordinate system at time $t_{1}$

$$
\dot{T}_{f_{t_{1, \text { slam }}}^{m}}^{m}=\left(\dot{X}_{f_{t_{1, \text { slam }}}^{m}}^{m} \quad \dot{Y}_{f_{t_{1, \text { slam }}}^{m}}^{m}, \quad \dot{Z}_{f_{t_{1, \text { slam }}}^{m}}^{m}\right)^{T}
$$

\subsubsection{Attitude}

The three attitude parameters can be determined by integrating time with the angular velocity. Since the IMU observes angular velocities in the IMU sensor system (s), they need to be rotated to the frame coordinate system (f) with the time-independent rotation matrix $R_{S}^{f}$.

$$
\left(\begin{array}{c}
\dot{\omega}_{i m u}^{f_{t_{i}}} \\
\dot{\varphi}_{i m u} \\
f_{t_{i}} \\
\dot{\kappa}_{i m u}
\end{array}\right)=R_{s}^{f}\left(\begin{array}{c}
\dot{\omega}_{i m u} s_{t_{i}} \\
\dot{\varphi}_{i m t_{i}} \\
\dot{\varphi}_{i m u} \\
\dot{\kappa}_{i m u} s_{t_{i}}
\end{array}\right)
$$

We obtain the incremental angles of the frame rotation from $t_{i}$ to $t_{i+1}$. If we multiply the angular velocities in the frame coordinate system by $\Delta t=t_{i+1}-t_{i}$, we arrive at:

$$
R_{f_{t_{i}}}^{f_{t_{i+1}}}=R_{1}\left(\dot{\omega}_{i m u}^{f_{t_{i}}} \Delta t\right) R_{2}\left(\dot{\varphi}_{i m u}^{f_{t_{i}}} \Delta t\right) R_{3}\left(\dot{\kappa}_{i m u}^{f_{t_{i}}} \Delta t\right)
$$

Then, the rotation matrix $R_{f_{t_{i+1}}}^{m}$ from the frame coordinate system to the model coordinate system at $t_{i+1}$ can be computed as follows:

$$
R_{f_{t_{i+1}}}^{m}=R_{f_{t_{i}}}^{m}\left(R_{f_{t_{i}}}^{f_{t_{i+1}}}\right)^{T}
$$

where $R_{f_{t_{i}}}^{m}=R_{f_{t_{1, \text { slam }}}^{m}}^{m}$, if $i=1$, and $R_{f_{t_{i}}}^{m}=R_{f_{t_{i-1}}}^{m}$ otherwise.

From the resulting rotation matrix $R_{f_{t_{i+1}}}^{m}$, we can infer the predicted attitude parameters (rotation angles) at $t_{i+1}, \omega_{f_{t_{i+1}}}^{m}$, $\varphi_{f_{t_{i+1}}}^{m}, \kappa_{f_{t_{i+1}}^{m}}^{m}$.

\subsubsection{Position}

The three position parameters can be derived via the double integration of the acceleration. The IMU also observes accelerations in its sensor system and can be rotated to the model coordinate system using the $R_{s}^{f}$ and $R_{f_{t_{i}}}^{m}$ predicted above as follows:

$$
\left(\begin{array}{l}
\ddot{X}_{i m u_{-} t_{i}}^{m} \\
\ddot{Y}_{i m u_{-} t_{i}}^{m} \\
\ddot{Z}_{\text {imu_t }}^{m} t_{i}
\end{array}\right)=R_{f_{t_{i}}^{m}}^{m} R_{s}^{f}\left(\begin{array}{c}
\ddot{X}_{i m u}^{s_{t_{i}}} \\
\ddot{Y}_{i m u}^{s_{t_{i}}} \\
\ddot{Z}_{i m u}^{s_{t_{i}}}
\end{array}\right)
$$

As the accelerations are now resolved in the model system where the $\mathrm{z}$-axis is assumed to be vertical, we subtract the average gravity $(g)$ from the acceleration along this axis.

$$
\ddot{T}_{i m u_{-} t_{i}}^{m}=\left(\begin{array}{c}
\ddot{X}_{i m u_{-} t_{i}}^{m} \\
\ddot{Y}_{i m u_{-} t_{i}}^{m} \\
\ddot{Z}_{i m u_{-} t_{i}}^{m}-g
\end{array}\right)
$$

Using these accelerations and the known frame position and velocity at $t_{i}$, we can obtain the frame position and velocity at $t_{i+1}$ as follows:

$$
T_{f_{t_{i+1}}}^{m}=T_{f_{t_{i}}}^{m}+\Delta t \dot{T}_{f_{t_{i}}}^{m}+\frac{1}{2} \Delta t^{2} \ddot{T}_{i m u_{-} t_{i}}^{m}
$$

$$
\dot{T}_{f_{t_{i+1}}}^{m}=\dot{T}_{f_{t_{i}}}^{m}+\Delta t \ddot{T}_{i m u_{-} t_{i}}^{m}
$$

where $\dot{T}_{f_{t_{i}}}^{m}=\dot{T}_{f_{t_{1, \text { slam }}}}^{m}$ and $T_{f_{t_{i}}}^{m}=T_{f_{t_{1, \text { slam }}}}^{m}$, if $i=1$, and $\dot{T}_{f_{t_{i}}}^{m}=\dot{T}_{f_{t_{i-1}}}^{m}$ and $T_{f_{t_{i}}}^{m}=T_{f_{t_{i-1}}}^{m}$ otherwise. 


\subsubsection{SLAM and IMU Integration}

The IMU fusion with the SLAM system is based mainly on the replacement of linear extrapolation by the IMU-based prediction, eq.(6) and eq.(10), to test the data association within the local window. Here, we fit cubic splines through all the predicted poses (six cubic splines for six pose parameters) and used these splines to test the data association.

\section{DATASETS}

Two datasets are captured by the backpack mapping system and used in this work. The first dataset is collected in a cube-shaped room with some bending movements. Specifically, the operator first stands inside a room with planar and vertical structures, which represents an optimal environment for our SLAM. Then the operator starts recording data with the scanners and IMU while bending forward and sideward (right and left), then rotating $90^{\circ}$ to perform these bends again. He continues the rotation and bending steps until he is back at his starting orientation. The dataset collected in this manner is then utilized for IMU data analysis, as described in Section 5.1.

The second dataset is acquired at the University of Braunschweig, Germany. The scanned floor shows a distinct office environment. It is the main dataset used in this study and is utilized to investigate the IMU prediction in comparison with linear extrapolation and show the benefits of the IMU-SLAM integration, as described in Sections 5.2 and 6.

\section{ANALYSIS OF IMU PERFORMANCE}

\subsection{IMU Data Analysis}

In order to analyse the IMU data, we check the consistency of the IMU angular velocity with the first-order derivatives of the rotation splines estimated by the laser-based SLAM. We run the SLAM on the first dataset because we have large changes in rotation around all three axes, and we want to check if the approximate values of rotational rates estimated by SLAM show the same pattern as the rotational rates measured by the IMU. The angular velocities $\left(\dot{\omega}_{i m u}^{\mathrm{s}}, \dot{\varphi}_{i m u}^{\mathrm{s}}, \dot{\kappa}_{i m u}^{\mathrm{s}}\right)$ measured by the IMU are observed around the axes of the IMU sensor. What we need are the partial derivatives of the rotation angles (splines) estimated by SLAM and used to rotate between the model and the frame coordinate system, i.e. $\dot{\omega}_{f, \text { slam }}^{m}, \quad \dot{\varphi}_{\mathrm{f}, \text { slam }}^{m}, \quad \dot{\kappa}_{f, \text { slam }}^{m}$. To determine the relationship with the observed angular velocities, we first need to define the order and direction of rotation exactly. So far, we have defined $R_{\mathrm{f}}^{m}$ using eq.(1); hence, the rotation from the model coordinate system to the frame coordinate system $R_{m}^{\mathrm{f}}$ can be defined as:

$$
R_{m}^{\mathrm{f}}=\left(R_{\mathrm{f}}^{m}\right)^{T}=R_{3}\left(\kappa_{f, \text { slam }}^{m}\right)^{T} R_{2}\left(\varphi_{f, \text { slam }}^{m}\right)^{T} R_{1}\left(\omega_{f, \text { slam }}^{m}\right)^{T}
$$

Once the model coordinate system is aligned with the frame coordinate system, we can apply the time-independent rotation $R_{f}^{S}=\left(R_{s}^{f}\right)^{T}$ from the frame to the IMU sensor coordinate system. Here, the entire rotation from the model coordinate system to the IMU sensor coordinate system will be:

$$
R_{m}^{s}=R_{f}^{S} R_{m}^{f}
$$

As $\kappa$ is the first rotation applied when rotating from the frame to the model coordinate system, we do not have to rotate the $\dot{\kappa}_{\text {slam }}^{m}$ from the model to the frame.
The measured angular velocity $\dot{\varphi}_{\text {imu }}^{\mathrm{S}}$ around the y-axis does not correspond directly to the first derivative of $\varphi_{f, s l a m}^{m}$ when just rotating $\left(R_{f}^{S}\right)$ from the frame to the IMU sensor coordinate system because the $y$-axis has already been rotated by $-\kappa_{f, \text { slam }}^{m}$ around the z-axis prior to measuring $\dot{\varphi}_{i m u}^{\mathrm{S}}$ in the IMU sensor coordinate system. Hence, the derivative of $\varphi_{f, \text { slam }}^{m}$ should also be rotated to the frame coordinate system. Similarly, the derivative of $\omega_{f, \text { slam }}^{m}$ needs to be rotated by $-\varphi_{f, \text { slam }}^{m}$ around the y-axis and $-\kappa_{f, \text { slam }}^{m}$ around the z-axis to obtain an angular velocity vector in the frame coordinate system. So, all the rotated angular velocity vectors together determine the angular rotation velocities that are measured in the frame coordinate system. Hence, after rotating from the frame to the IMU coordinate system, we obtain the angular velocities as derived by the SLAM in the IMU coordinate system as

$$
\begin{gathered}
\left(\begin{array}{c}
\dot{\omega}_{\text {slam }}^{S} \\
\dot{\varphi}_{\text {slam }}^{S} \\
\dot{\kappa}_{\text {slam }}^{S}
\end{array}\right)=R_{f}^{S}\left(\begin{array}{c}
0 \\
0 \\
\dot{\kappa}_{f, \text { slam }}^{m}
\end{array}\right)+R_{f}^{S} R_{3}\left(\kappa_{f, \text { slam }}^{m}\right)^{T}\left(\begin{array}{c}
0 \\
\dot{\varphi}_{\mathrm{f}, \text { slam }}^{m} \\
0
\end{array}\right)+ \\
R_{f}^{S} R_{3}\left(\kappa_{f, \text { slam }}^{m}\right)^{T} R_{2}\left(\varphi_{f, \text { slam }}^{m}\right)^{T}\left(\begin{array}{c}
\dot{\omega}_{f, \text { slam }}^{\mathrm{m}} \\
0 \\
0
\end{array}\right)
\end{gathered}
$$

These angular velocities should be comparable to those measured by the IMU. To verify this, the angular velocities $\left(\dot{\omega}_{\text {slam }}^{\mathrm{s}}, \dot{\varphi}_{\text {slam }}^{\mathrm{s}}, \dot{\kappa}_{\text {slam }}^{\mathrm{s}}\right)$ are plotted against $\left(\dot{\omega}_{\text {imu }}^{\mathrm{s}}, \dot{\varphi}_{\text {imu }}^{\mathrm{s}}, \dot{\kappa}_{\text {imu }}^{\mathrm{s}}\right)$ and the differences are computed. Figure 2 shows $\dot{\varphi}_{f, \text { slam }}^{S}$ plotted against $\dot{\varphi}_{i m u}^{S}$. After the burn-off period of $0.2 \mathrm{~s}$, the results are promising because both the IMU- and SLAM- based angular velocities exhibit the same patterns. The differences between the IMU and SLAM angular velocities along all axes are mostly within $\pm 0.05-0.1$ degrees/s.

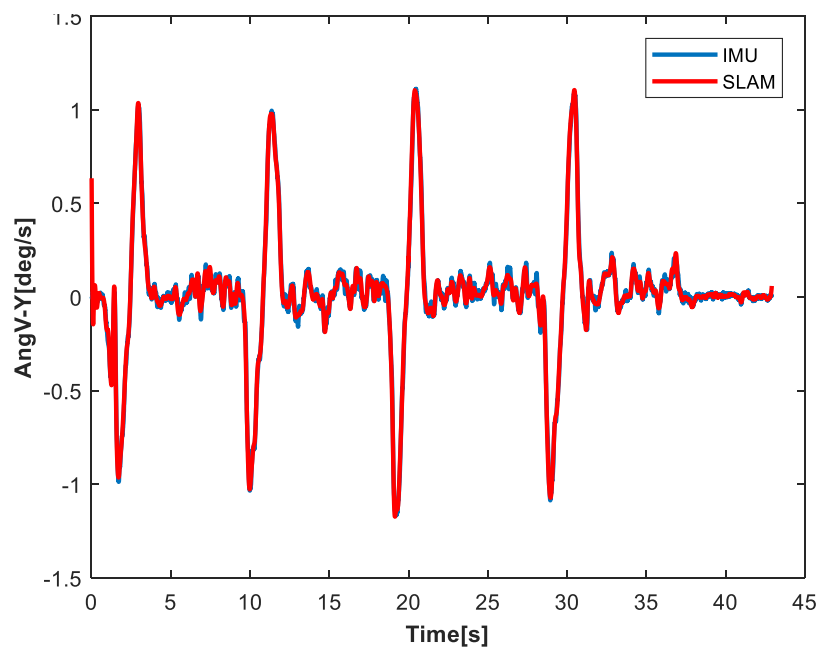

Figure 2. An example plot for testing the IMU integration. The angular velocities measured by the IMU along the y-axis are plotted against the first-order derivatives of the rotation y-splines estimated by the laser-based SLAM. Similar tests were done for all axes of the gyroscope.

\subsection{IMU Prediction Analysis}

We run the laser-based SLAM on the second dataset. As an initial analysis of the SLAM-IMU combined performance, we use the IMU data to predict the pose of the next scan $\left(P_{\text {pred }}^{\text {imu }}\right)$ using the equations introduced in Section 3.4 and compare the resulting pose with the linearly predicted one $\left(P_{\text {pred }}^{\text {linear }}\right)$. As ground truth we use the poses $\left(P_{\text {est }}\right)$ obtained in the optimal global pose 
estimation of the SLAM procedure. Figure 3 shows that the predicted rotation angles around the $\mathrm{X}$-axis $(\omega)$ and $\mathrm{Z}$-axis $(\kappa)$ by the IMU are closer to the ground truth angles than the linearly predicted ones. Table 1 lists the RMSE values computed using eq.(14) for all the pose parameters, namely, the rotation angles $(\omega, \phi, \kappa)$ and positions $(\mathrm{X}, \mathrm{Y}, \mathrm{Z})$.

$$
\operatorname{RMSE}_{P}^{\text {meth }}=\sqrt{\frac{1}{N} \sum_{i=1}^{N}\left(P_{\text {pred } \_i}^{\text {meth }}-P_{\text {est } \_i}\right)^{2}}
$$

where $\mathrm{P}$ refers to one the pose parameters $\{\mathrm{x}, \mathrm{y}, \mathrm{z}, \omega, \varphi, \kappa\}, P_{\text {est }}$ is the estimated pose parameter by the laser-based SLAM. The term "meth" refers to the prediction method, "imu" when we rely on IMU for pose prediction and "linear" otherwise.

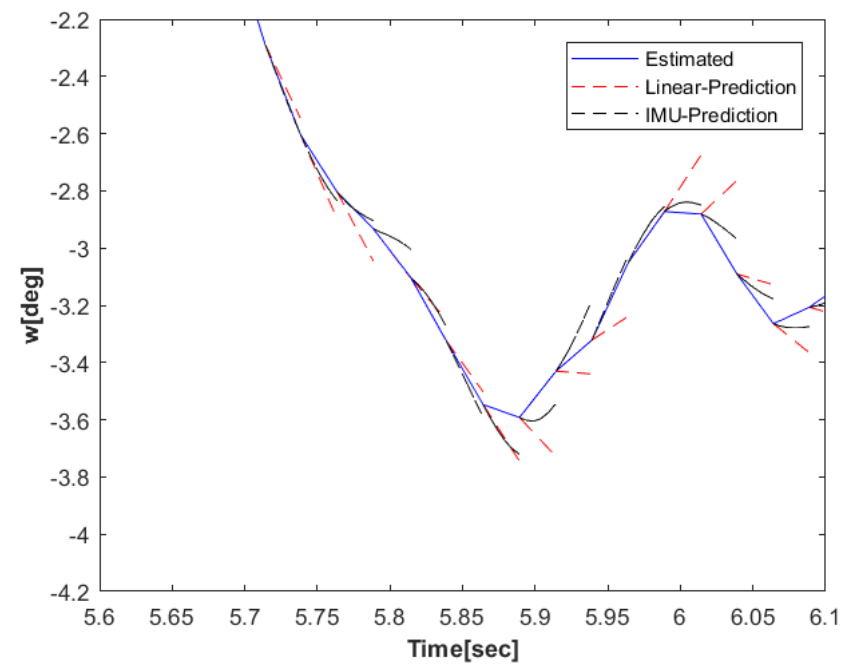

(a)

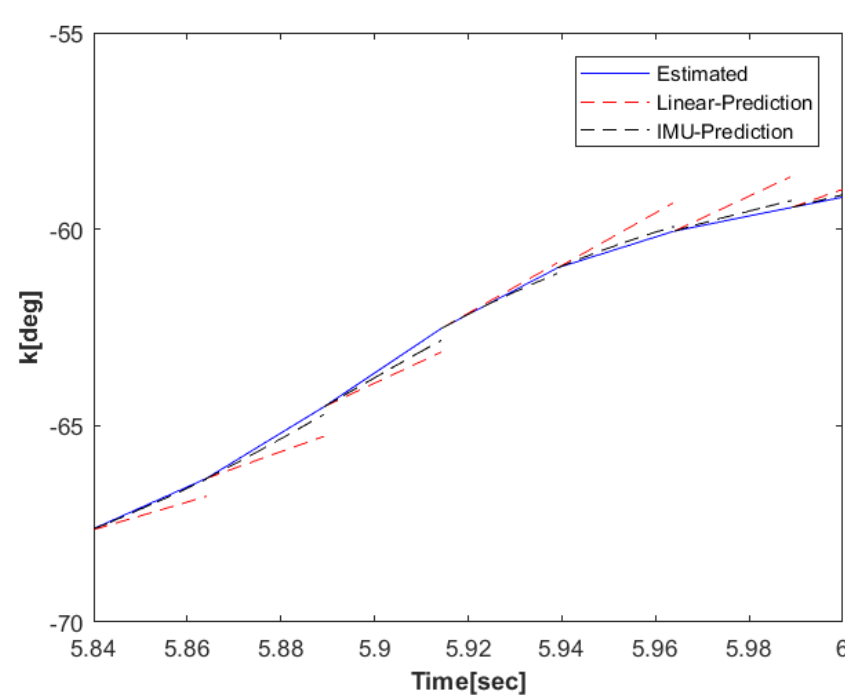

(b)

Figure 3. Part of the rotation angles $(\omega, \kappa)$ trajectories. (a) for $\omega$. (b) for $\kappa$. Each blue line connects the two estimated angles ( $\omega$,

$\kappa)$ at the start and end of one local window $(\approx 25 \mathrm{~ms})$. The dashed black and red lines are the IMU and linear predictions of angle $(\omega, \kappa)$, respectively.

\begin{tabular}{|c|c|c|c|c|c|c|}
\hline \multirow{2}{*}{ SLAM } & \multicolumn{3}{|c|}{$\begin{array}{c}\text { Rotation Angles } \\
\text { RMSE (deg) }\end{array}$} & \multicolumn{3}{c|}{ Position RMSE (m) } \\
\cline { 2 - 7 } & $\omega$ & $\varphi$ & $\kappa$ & $\mathrm{X}$ & $\mathrm{Y}$ & $\mathrm{Z}$ \\
\hline $\begin{array}{c}\text { With } \\
\text { IMU }\end{array}$ & 0.092 & 0.106 & 0.100 & 0.0054 & 0.0033 & 0.0031 \\
\hline $\begin{array}{c}\text { Without } \\
\text { IMU }\end{array}$ & 0.166 & 0.160 & 0.231 & 0.0054 & 0.0032 & 0.0032 \\
\hline
\end{tabular}

Table 1. RMSE values of all the predicted pose parameters, rotation angles, and position coordinates.

The RMSE results indicate that the IMU prediction of orientation is about two times more accurate than the linear prediction. As the operator walking speed is usually less than $1.4 \mathrm{~m} / \mathrm{s}$, the expected translation between two scanlines (within $25 \mathrm{~ms}$ ) is about $3 \mathrm{~cm}$. Thus, the fractions of millimetres improvement in position prediction is irrelevant for data association. The most crucial issue is the orientation prediction because the operator can make a large change in orientation within $25 \mathrm{~ms}$ or an even shorter time span. Moreover, a small error in the orientation prediction can have serious effects on the data association quality. As a simple example, if there is a point at a distance of $10 \mathrm{~m}$ from the system, a linear prediction error of the angle $\kappa$ of one sigma $\left(0.231^{\circ}\right)$ would already result in a $4.0 \mathrm{~cm}$ lateral displacement of this point compared to a $1.7 \mathrm{~cm}$ lateral displacement with the IMU prediction.

\section{INTEGRATION RESULTS AND DISCUSSION}

In order to test the performance of the IMU-SLAM integration, the IMU prediction-based SLAM was run on the second dataset. The generated point cloud is shown in Figure 5. In order to evaluate the benefits of this integration, we compare the number of points assigned to the planes and the RMSE of the residuals in two cases: SLAM with and without IMU prediction (Table 2). In this paper, SLAM without IMU refers to the linear predictionbased SLAM. The residuals are the distances of the points to the estimated planes.

\begin{tabular}{|c|c|c|}
\hline SLAM & Without IMU & With IMU \\
\hline Number of assigned points & 24527978 & 24562619 \\
\hline RMSE of the residuals $(\mathrm{cm})$ & 1.80 & 1.31 \\
\hline
\end{tabular}

Table 2. The number of assigned points and the corresponding RMSEs of the residuals.

The table shows that using the IMU data to predict the pose of the next scans slightly increases the number of points assigned to the planes. More importantly, the RMSE value of the points' residuals with IMU prediction is quite a lot lower than that obtained via linear prediction, meaning that there are more correct associations when the IMU is used for pose prediction.

In addition, histograms of the computed distances (residuals) between the assigned points and their corresponding reconstructed planes are generated in order to provide an overall impression of the data association quality in both cases. Each histogram is built with $0.01 \mathrm{~m}$ bins, as shown in Figure 4, demonstrating that approximately $13 \%$ of the residuals exceed 3 $\mathrm{cm}$ when the IMU is not used. This percentage decreases to less than $4 \%$ when the IMU is utilized in pose prediction. 


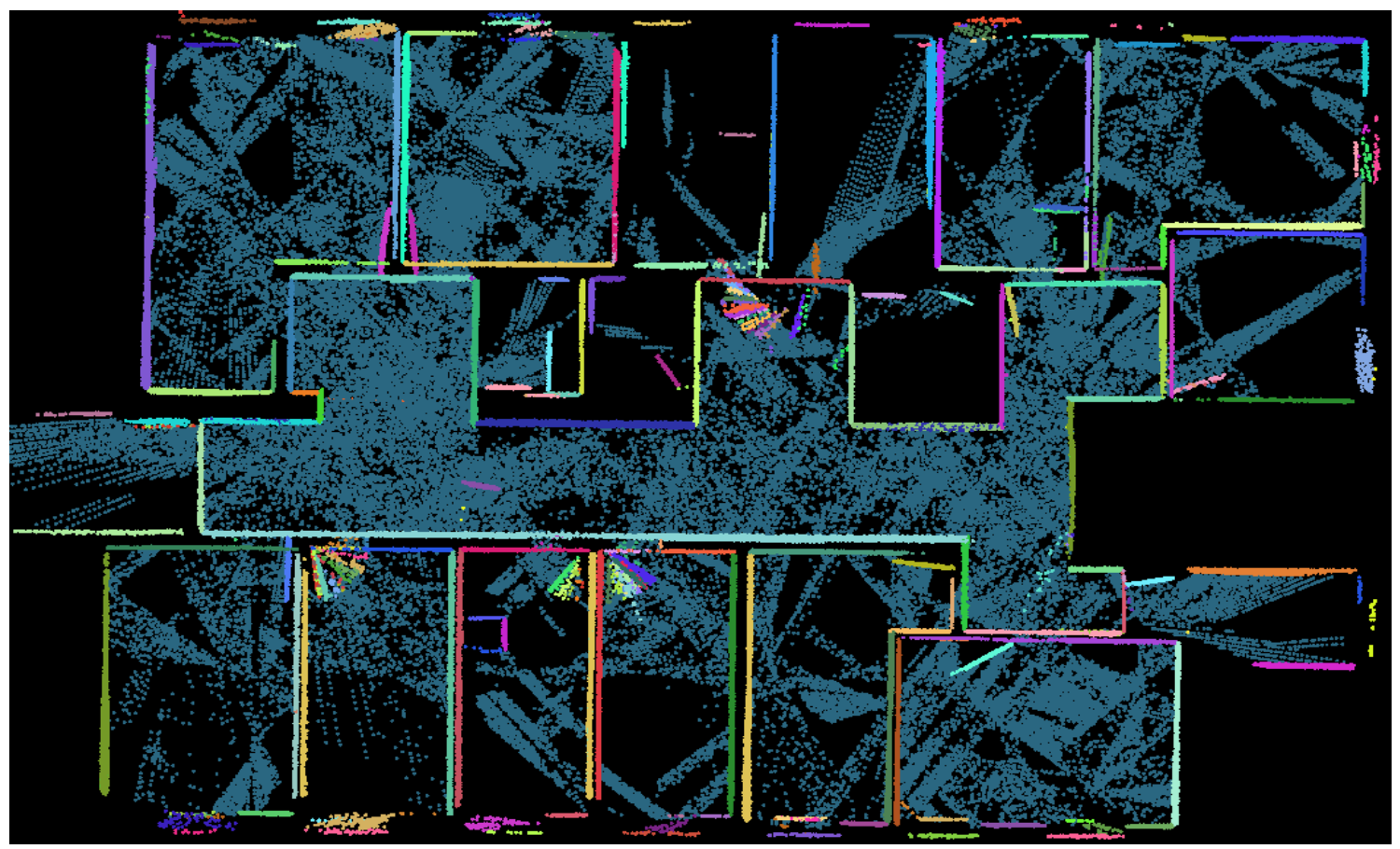

Figure 5. A top view of the generated point cloud by SLAM with IMU. The colours indicate point associations to a particular plane.

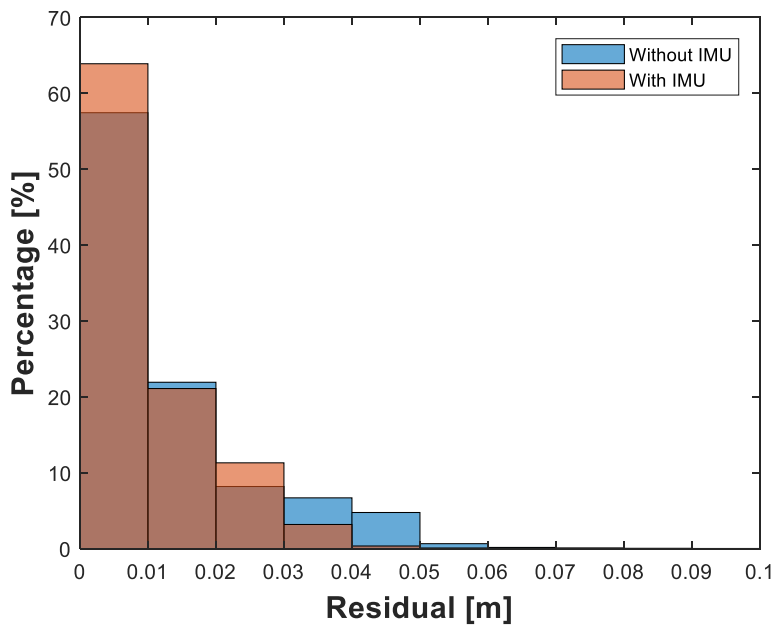

Figure 4. Histograms of the points' residuals in two cases, SLAM with and without IMU.

Since the perpendicularity and parallelism characteristics are predominant in the second indoor environment we scanned, we utilize our evaluation method with architectural constraints (Karam et al., 2018) to evaluate the ability of our updated mapping system to capture the true geometry of its environment. The method is applied with same thresholds on the reconstructed planes by SLAM in two cases, with and without IMU. We compute the angles between the (perpendicular/parallel) reconstructed planes and we derive the deviations of these angles from the corresponding expected value $\left(90^{\circ} / 0^{\circ}\right)$. The computed deviations are called angles' errors. The results of this evaluation process are summarized in tables $(3,4)$.

\begin{tabular}{|c|c|c|c|c|}
\hline SLAM & Errors range & {$\left[0^{\circ} 0.5^{\circ}[\right.$} & {$\left[0.5^{\circ} 1^{\circ}\right]$} & $>1^{\circ}$ \\
\hline \multirow{2}{*}{ With } & parallelism & $66 \%$ & $13 \%$ & $21 \%$ \\
\cline { 2 - 5 } IMU & perpendicularity & $70 \%$ & $23 \%$ & $7 \%$ \\
\hline \multirow{2}{*}{$\begin{array}{c}\text { Without } \\
\text { IMU }\end{array}$} & parallelism & $50 \%$ & $12 \%$ & $38 \%$ \\
\cline { 2 - 5 } & perpendicularity & $58 \%$ & $26 \%$ & $16 \%$ \\
\hline
\end{tabular}

Table 3. Percentages of angles' errors for parallelism and perpendicularity in two cases, SLAM with and without IMU.

\begin{tabular}{|c|c|c|}
\hline SLAM & Constraint & RMSE \\
\hline \multirow{2}{*}{$\begin{array}{l}\text { With } \\
\text { IMU }\end{array}$} & parallelism & $1.39^{\circ}$ \\
\cline { 2 - 3 } & perpendicularity & $0.66^{\circ}$ \\
\hline \multirow{2}{*}{$\begin{array}{c}\text { Without } \\
\text { IMU }\end{array}$} & parallelism & $1.76^{\circ}$ \\
\cline { 2 - 3 } & perpendicularity & $1.34^{\circ}$ \\
\hline
\end{tabular}

Table 4. RMSE of angles' errors for parallelism and perpendicularity in two cases, SLAM with and without IMU.

The results show that IMU-based prediction improves the reconstruction accuracy where the percentages of small angles' errors $\left(<0.5^{\circ}\right)$ increases and of outliers $\left(>1^{\circ}\right)$ decreases.

The level of improvements addressed above is linked directly to the IMU drift rate specifications. In other words, if the IMU performance is efficient, larger and more sudden rotations can be handled. Hence, we predict the orientation and translation of the system using available IMU data within a local window. In this study, the width of this local window is selected to cover the time interval of one scanline.

In our previous work, we compensated for the low pose update frequency by using overly-relaxed data association thresholds (Karam et al., 2019). Now, with the IMU prediction, these thresholds can be tightened to create a more robust data association process. Consequently, the pose estimation and the output planar representation become more accurate. 


\section{CONCLUSIONS AND FUTURE WORK}

In this paper, we presented an improvement of our laser-based SLAM algorithm by integrating an IMU sensor. We show that even a low-cost IMU improves the accuracy of the predicted pose within SLAM. Furthermore, this improves also the robustness of the data association.

In future work, we intend to deepen the IMU integration by including the IMU observations in the pose estimation equations. More observations might enable us to use cubic instead of linear splines and increase the robustness in the estimation process. Moreover, we plan to test how long we can rely on the IMU for prediction. Reliable IMU prediction for a wider local window could lead into a better hypothesis generation for the planar structures, thus this enabling the SLAM to work in a more complex environment (e.g. with slanted walls).

\section{REFERENCES}

Ajay Kumar, G., Patil, A.K., Patil, R., Park, S.S., Chai, Y.H., 2017. A LiDAR and IMU integrated indoor navigation system for UAVs and its application in real-time pipeline classification. Sensors (Switzerland) 17. doi.org/10.3390/s17061268.

Blaser, S., Nebiker, S., Wisler, D., 2019. Portable image-based high performance mobile mapping system in underground environments - system configuration and performance evaluation. ISPRS Ann. Photogramm. Remote Sens. Spat. Inf. Sci. 4, 255-262. doi.org/10.5194/isprs-annals-IV-2-W5-255-2019.

Bosse, M., Zlot, R., Flick, P., 2012. Zebedee: Design of a springmounted 3-D range sensor with application to mobile mapping. IEEE Trans. Robot. 28, 1104-1119.

Celik, K., Soon-Jo Chung, Somani, A., 2008. Mono-vision corner SLAM for indoor navigation. In IEEE International Conference on Electro/Information Technology. IEEE, pp. 343348. doi.org/10.1109/EIT.2008.4554326.

Chow, J.C.K., Lichti, D.D., Hol, J.D., Bellusci, G., Luinge, H., 2014. IMU and multiple RGB-D camera fusion for assisting indoor stop-and-go 3D terrestrial laser scanning. Robotics 3, 247-280. doi.org/10.3390/robotics3030247.

Concha, A., Loianno, G., Kumar, V., Civera, J., 2016. Visualinertial direct SLAM. In IEEE International Conference on Robotics and Automation (ICRA). IEEE, pp. 1331-1338. doi.org/10.1109/ICRA.2016.7487266.

Davison, A. J., Reid, I. D., Molton, N. D., \& Stasse, O., 2007. MonoSLAM: Real-time single camera SLAM. IEEE Trans. pattern Anal. Mach. Intell. 29.6 (2007). 29, 1052-1067.

Durrant-Whyte, H., Bailey, T., 2006a. Simultaneous localization and mapping (SLAM): part I The Essential Algorithms. Robot. Autom. Mag. 2, 99-110.

Durrant-Whyte, H., Bailey, T., 2006b. Simultaneous Localization and Mapping (SLAM): Part II. IEEE Robot. Autom. Mag. 13.3 108-117.

García, S., López, M.E., Barea, R., Bergasa, L.M., Gómez, A., Molinos, E.J., 2016. Indoor SLAM for micro aerial vehicles control using monocular camera and sensor fusion. Proc. - 2016 Int. Conf. Auton. Robot Syst. Compet. ICARSC 2016 205-210. doi.org/10.1109/ICARSC.2016.46.
GeoSLAM Ltd. The ZEB-REVO Solution. 2018. Available online: https://geoslam.com/solutions/zeb-revo/ (accessed on 20 October 2019).

Grisetti, G., Stachniss, C., Burgard, W., 2007. Improved Techniques for Grid Mapping. Robot. IEEE Trans. 23, 34-46.

Henry, P., Krainin, M., Herbst, E., Ren, X., Fox, D., 2014. RGBD Mapping: Using Depth Cameras for Dense 3D Modeling of Indoor Environments. In: Experimental Robotics, Springer Tracts in Advanced Robotics, Vol. 79. pp. 477-491. doi.org /10.1007/978-3-642-28572-1_33.

Hyyti, H., Visala, A., 2015. A DCM Based Attitude Estimation Algorithm for Low-Cost MEMS IMUs. Int. J. Navig. Obs. 2015. doi.org/10.1155/2015/503814

Karam, S., Peter, M., Hosseinyalamdary, S., Vosselman, G., 2018. An Evaluation Pipeline for Indoor Laser Scanning Point Clouds. ISPRS Ann. Photogramm. Remote Sens. Spat. Inf. Sci. IV-1, 85-92. doi.org/10.5194/isprs-annals-IV-1-85-2018.

Karam, S., Vosselman, G., Peter, M., Hosseinyalamdary, S., Lehtola, V., 2019. Design, calibration, and evaluation of a backpack indoor mobile mapping system. Remote Sens. 11 doi.org/10.3390/rs11080978.

Lauterbach, H.A., Borrmann, D., Heß, R., Eck, D., Schilling, K., Nüchter, A., 2015. Evaluation of a backpack-mounted 3D mobile scanning system. Remote Sens. 7, 13753-13781. doi.org /10.3390/rs71013753.

Lehtola, V. V., Kaartinen, H., Nüchter, A., Kaijaluoto, R., Kukko, A., Litkey, P., Honkavaara, E., Rosnell, T., Vaaja, M.T., Virtanen, J.P., Kurkela, M., El Issaoui, A., Zhu, L., Jaakkola, A., Hyyppä, J., 2017. Comparison of the selected state-of-the-art 3D indoor scanning and point cloud generation methods. Remote Sens. 9, 1-26. doi.org/10.3390/rs9080796.

Lehtola, V. V., Virtanen, J.-P., Vaaja, M.T., Hyyppä, H., Nüchter, A., 2016. Localization of a mobile laser scanner via dimensional reduction. ISPRS J. Photogramm. Remote Sens. 121, 48-59. doi.org/10.1016/J.ISPRSJPRS.2016.09.004.

Leica Geosystems AG. Leica Pegasus: Backpack, Mobile Reality Capture. Available online: https://www.gefos-leica.cz/data/ original/skenery/mobilni-apovani/backpack/leica pegasusbackpack_ds.pdf (accessed on 18 October 2019).

Leutenegger, S., Furgale, P., Rabaud, V., Chli, M., Konolige, K., Siegwart, R., 2015. Keyframe-Based Visual-Inertial SLAM Using. Int. J. Rob. Res. 34, 314-334.

Liu, T.; Carlberg, M.; Chen, G.; Chen, J.; Kua, J.; Zakhor, A., 2010. Indoor localization and visualization using a humanoperated backpack system. Int. Conf. Indoor Position. Indoor Navig. (IPIN), 15-17 Sept. 2010, Zurich, Switz. 15-17.

Makni, A., Fourati, H., Kibangou, A.Y., 2014. Adaptive Kalman filter for MEMS-IMU based attitude estimation under external acceleration and parsimonious use of gyroscopes. Eur. Control Conf. ECC 2014 1379-1384. doi.org/10.1109/ ECC.2014.6862535.

Maximov, V., 2013. Survey of Accuracy Improvement Approaches for Tightly Coupled ToA / IMU Personal Indoor 
Navigation System. Proc. Int. Conf. Indoor Position. Indoor Navig. Oct. 2013, Montbeliard, Fr.

Naikal, N., Kua, J., Chen, G., Zakhor, A., 2009. Image Augmented Laser Scan Matching for Indoor Dead Reckoning. Proc. IEEE/RSJ Int. Conf. Intell. Robot. Syst. 4134-4141..

Norris, J., 2013. Future Trends in Geospatial Information Management: the five to ten year vision. Ordnance Surv. Req. Secr. United Nations Comm. Expert. Glob. Geospatial Inf. Manag. (UN-GGIM). Second Ed. December (2015).

Raúl Feliz, Eduardo Zalama, J.G.G.-B., 2009. Pedestrian tracking using inertial sensors. J. Phys. AGENTS 3, 35.

S. Kohlbrecher, J. Meyer, O. Von Stryk, U.K., 2011. A Flexible and Scalable SLAM System with Full 3D Motion Estimation. Int. Symp. Safety, Secur. Rescue Robot.

Velas, M., Spanel, M., Sleziak, T., Habrovec, J., Herout, A., 2019. Indoor and Outdoor Backpack Mapping with Calibrated Pair of Velodyne LiDARs. Sensors 19, 3944. doi.org /10.3390 /s19183944

Vosselman, G., 2014. Design of an indoor mapping system using three 2D laser scanners and 6 DOF SLAM. ISPRS Ann. Photogramm. Remote Sens. Spat. Inf. Sci. 2.3173.

Wang, Z., Zhu, H., Zhou, J., Wang, X., 2018. Loose fusion based on SLAM and IMU for indoor environment. In: Yu, H., Dong, J. (Eds.), Ninth International Conference on Graphic and Image Processing (ICGIP 2017). SPIE, p. 97. doi.org/10.1117 $/ 12.2302929$.

Wen, C., Pan, S., Wang, C., Li, J., 2016. An Indoor Backpack System for 2-D and 3-D Mapping of Building Interiors. IEEE Geosci. Remote Sens. Lett. 13, 992-996. 